\title{
Selective suppression of Dresselhaus or Rashba spin-orbit coupling effects by the Zeeman interaction in quantum dots
}

\author{
B. Szafran, ${ }^{1}$ M. P. Nowak, ${ }^{1}$ S. Bednarek, ${ }^{1}$ T. Chwiej, ${ }^{1}$ and F. M. Peeters ${ }^{2}$ \\ ${ }^{1}$ Faculty of Physics and Applied Computer Science, AGH University of Science and Technology, al. Mickiewicza 30, \\ 30-059 Kraków, Poland \\ ${ }^{2}$ Departement Fysica, Universiteit Antwerpen, Groenenborgerlaan 171, B-2020 Antwerpen, Belgium
}

(Received 17 March 2009; revised manuscript received 21 April 2009; published 2 June 2009)

\begin{abstract}
We study single- and two-electron parabolic quantum dots in the presence of linear Dresselhaus and Rashba spin-orbit interactions. Contributions of both types of spin-orbit coupling are investigated in the context of the spin polarization of the system at high magnetic fields. We demonstrate that for negative Landé factors the effect of the Dresselhaus coupling is suppressed at high magnetic field, which for structures without inversion asymmetry leads to a completely spin-polarized system and a strict antisymmetry of the wave functions with respect to the interchange of spatial-electron coordinates. For negative Landé factor the Rashba coupling is preserved at high field and consequently the spin polarization of the systems as well as the spatial antisymmetry of the two-electron wave function remain approximate.
\end{abstract}

DOI: 10.1103/PhysRevB.79.235303

PACS number(s): 73.40.Gk

\section{INTRODUCTION}

In quasi-two-dimensional semiconductor systems inversion asymmetry of the potential profile in the growth direction introduces spin-orbit (SO) interaction for the confined carriers known as structure-asymmetry-induced or Rashba coupling. ${ }^{1}$ Moreover, in III-V's and II-VI's the inversion asymmetry of the crystal lattice introduces SO coupling of the Dresselhaus ${ }^{2}$ type. Coupling of the spin and orbital degrees of freedom is an important issue for quantum information processing applications based on spins of electrons confined in quantum dots. ${ }^{3}$ In particular, the SO coupling can be used to accompany the orbital electron movement by the rotations of its spin. ${ }^{4,5}$ The SO coupling allows also for spin rotations of localized electrons subject to oscillating electric fields. ${ }^{6}$ In the context of the two-qubit operations the SO coupling introduces anisotropy in the exchange interaction ${ }^{7,8}$ which allows for the construction of universal quantum gates without single-spin rotations. ${ }^{8}$

The initial and final states of a quantum gate should preferably correspond to quantum-dot-confined stationary states with a definite spin orientation. However, the SO coupling leads to decay of the spin polarization. ${ }^{9}$ For two-electron quantum dots this amounts in the triplet-singlet relaxation. ${ }^{10}$ The relaxation of the spin-polarization results from the fact that in the presence of the SO coupling no component of the spin is a good quantum number. In other words in stationary dot-confined states the spin orientation can be only approximately but not strictly defined. The effect of the SO coupling for stationary states was studied in a number of theoretical papers, ${ }^{10-16}$ which (except the issues mentioned above) analyzed opening of avoided crossings between energy levels of definite spin and orbital momenta and their consequences for the optical spectrum or magnetization of the system.

In transport experiments ${ }^{17}$ which probe the ground-state properties of confined systems, usually both types of SO coupling are present and the separation of their relative contribution is difficult. ${ }^{18}$ The purpose of the present paper is to establish the role of both types of SO coupling in the context of the ground-state spin polarization at high magnetic field and the separability of spin and spatial degrees of freedom. A special attention is paid to the Zeeman effect which favors one of the spin orientations. The spin-orbit coupling effects should be completely suppressed provided that the Zeeman interaction perfectly polarizes the confined spins. The main message of the present work is that at high magnetic field for the Landé factor $g<0$ the effect of the Dresselhaus coupling is suppressed by the Zeeman interaction but the effect of the Rashba coupling is preserved.

Quantum dot properties at high magnetic field and in the absence of the SO coupling are often described in the approximation of spinless (spin-polarized) electrons ${ }^{19-23}$ with wave functions that are strictly antisymmetric with respect to the exchange of the electron spatial coordinates. In quantum dots the SO coupling - when present-excludes complete spin polarization of the electron system and conversely the complete spin polarization may be achieved only at the expense of the suppression of the SO effects. We show below that for $g<0$ at high field (i) for systems without structural inversion asymmetry the spin-up polarization of the ground state becomes complete and consequently, (ii) the wave function becomes strictly antisymmetric with respect to exchange of the electron spatial coordinates, and (iii) the Rashba coupling is preserved at high field and consequently for structures with inversion asymmetry the spin polarization remains incomplete and the spatial wave-function antisymmetry is only approximate.

This paper is organized in the following way. The theory is given in the second section. Sections III and IV contain results for one and two electrons. Summary and conclusions are provided in Sec. V.

\section{THEORY}

We consider the effective mass single-electron Hamiltonian of the form 


$$
H=h_{0}+H_{R}+H_{D},
$$

where $h_{0}$ stands for the Hamiltonian of the electron in a two-dimensional parabolic quantum dot for perpendicular magnetic-field $B$ without the SO coupling

$$
h_{0}=\left(\frac{\mathbf{p}^{2}}{2 m^{*}}+\frac{m^{*} \omega^{2} \mathbf{r}^{2}}{2}\right) \mathbf{1}+\frac{1}{2} g \mu_{B} B \sigma_{z},
$$

with $\mathbf{1}$-the $2 \times 2$ identity matrix and $\mathbf{p}=-i \hbar \nabla+e \mathbf{A}$, where $e>0$ is the elementary charge. The symmetric gauge $\mathbf{A}$ $=\frac{B}{2}(-y, x, 0)$ and GaAs electron effective mass $m^{*}=0.063 m_{0}$ are applied. $\mu_{B}$ the Bohr magneton and $\sigma$ 's stand for the Pauli matrices. We consider parabolic quantum dot confinement with $\hbar \omega=2 \mathrm{meV}$. The applied confinement corresponds to large quantum dots with lateral confinement potential of an electrostatic origin. ${ }^{24}$ term

In Eq. (1) $H_{R}\left(H_{D}\right)$ is the Rashba (Dresselhaus) coupling

$$
\begin{aligned}
& H_{R}=\alpha\left(\sigma_{x} p_{y}-\sigma_{y} p_{x}\right) / \hbar, \\
& H_{D}=\beta\left(\sigma_{x} p_{x}-\sigma_{y} p_{y}\right) / \hbar,
\end{aligned}
$$

where $\alpha$ and $\beta$ are the Rashba and Dresselhaus constants. In this paper we neglect the cubic contribution to the Dresselhaus coupling which for two-dimensional closed quantum dots produces small effects ${ }^{25}$ as compared to the linear Dresselhaus and Rashba terms. Linear Dresselhaus coupling constant for the two-dimensional SO interaction can be deduced from the bulk coupling constant $\gamma$ and the height of the quantum dot $d \beta=\left(\frac{\pi}{d}\right)^{2} \gamma$. For GaAs coupling constant ${ }^{26}$ $\gamma=27.5 \mathrm{eV} \AA^{3}$ and $d=5 \mathrm{~nm}$ one obtains $\beta=10.8 \mathrm{meV} \mathrm{nm}$. In quantum dots the Rashba coupling constant - that is proportional to the electric field in the growth direction-may achieve values similar to the Dresselhaus constant. ${ }^{18}$ The results of the present paper were obtained for coupling constants equal or smaller than $10.8 \mathrm{meV} \mathrm{nm}$.

Below we consider mainly the case of pure Dresselhaus coupling as well as pure Rashba coupling and the case of equal coupling constants. In real structures usually the coupling constants are not equal. For both couplings present the Zeeman effect at high magnetic field leaves unaffected one of the coupling types and suppresses the other irrespective of the relative values of the two SO coupling constants. Therefore, the results for the suppression of one of the SO coupling types by the Zeeman effect presented below for equal coupling constants are quite typical. On the other hand the case of equal coupling constant in the absence of the magnetic field are of a particular interest for two-dimensional quantum wells in which persistent spin-helix states were predicted to $\operatorname{appear}^{27}$ with infinite spin-relaxation times. The persistent helix states were recently detected in an experiment $^{28}$ in which the Rashba coupling constant was tuned to match the Dresselhaus constant with a voltage applied to a gate.

For structures built of GaAs/AlGaAs quantum wells the value of $g$ factor may be tuned by the width of the quantum well, ${ }^{29}$ in particular $g$ may be set to zero or positive values of $g$ can also be achieved. In the present paper we consider two cases $g=0$ and $g=-0.44$ (bulk GaAs Landé factor). Results for positive $g=+0.44$ can be obtained from the ones presented below for $g=-0.44$ when one exchanges the coupling constants $\alpha \rightleftharpoons \beta$ and inverts the spin direction $\sigma_{z} \rightleftharpoons-\sigma_{z}$.

For pure Rashba coupling $(\beta=0)$ and confinement potential of circular symmetry, Hamiltonian (1) commutes with the total angular momentum operator $J_{+}=L_{z}+S_{z}$, where $S_{z}$ $=\frac{\hbar}{2} \sigma_{z}$ is the operator of the $z$ component of the spin and $\mathbf{L}$ $=-i \hbar \mathbf{1}(\mathbf{r} \times \nabla)$ is the operator of the orbital angular momentum. For pure Dresselhaus coupling good quantum numbers are the eigenvalues of the total angular momentum defined with an opposite contribution of the spin $J_{-}=L_{z}-S_{z}$. In the following when discussing the eigenstates for a single type of SO coupling we will skip subscripts for $J_{+}$and $J_{-}$eigenvalues. For pure Rashba coupling as well as for pure Dresselhaus coupling both spin-up and spin-down wave-function components have definite orbital angular momentum

$$
\psi=\left(\begin{array}{c}
\psi_{l_{\uparrow}}(r) \exp \left(i l_{\uparrow} p h i\right) \\
\psi_{l_{\downarrow}}(r) \exp \left(i l_{\downarrow} \phi\right)
\end{array}\right)
$$

with $l_{\downarrow}=l_{\uparrow}+1$ for the Rashba and $l_{\downarrow}=l_{\uparrow}-1$ for the Dresselhaus coupling. When only a single type of SO coupling is present it is convenient to put Hamiltonian (1) in cylindrical coordinates and make use of the definite values of the orbital angular momentum for both components to separate the radial and angular degrees of freedom. Then one obtains a couple of radial eigenequations for each spin component to be solved. ${ }^{11-13,30}$

For both types of SO coupling present the components of the single-electron wave functions are no longer eigenstates of the orbital angular momentum. However, each of the wave-function components still has a definite parity with respect to point inversion, i.e., both components are eigenstates of the parity operator $P$ [defined as $P f(\mathbf{r})=f(-\mathbf{r})$, where $f$ is a scalar function]. This is because Hamiltonian (1) commutes with $P_{s}=P \sigma_{z}$, which we will refer to as the $s$-parity operator. The parities of the spin-up and spin-down components are opposite. Below we refer to the eigenstates of $P_{s}$ corresponding to the eigenvalue $+1(-1)$ as even (odd) $s$-parity states.

We solved the single-electron problem in the basis

$$
\psi_{\mu}=\left(\begin{array}{c}
\psi_{\mu}^{\uparrow} \\
\psi_{\mu}^{\downarrow}
\end{array}\right)=\sum_{n l} c_{n l}^{\mu \uparrow}\left(\begin{array}{c}
\psi_{n l} \\
0
\end{array}\right)+\sum_{n l} c_{n l}^{\mu \downarrow}\left(\begin{array}{c}
0 \\
\psi_{n l}
\end{array}\right),
$$

where $\psi_{n l}$ are the eigenfunctions of the $h_{0}$ operator, i.e., the Fock-Darwin (FD) states

$$
\begin{aligned}
\psi_{n l}= & \sqrt{\frac{n !}{\pi(n+|l|) !}}\left(\frac{1}{l_{B}}\right)^{|l|+1} r^{|l|} \exp (i l \phi) \\
& \times \exp \left(-r^{2} / 2 l_{B}^{2}\right) L_{n}^{|l|}\left(\frac{r^{2}}{l_{B}^{2}}\right),
\end{aligned}
$$

with $L_{n}^{l}$-the Laguerre polynomials and $l_{B}=\sqrt{\hbar / m^{*} \omega_{h}}$, corresponding to $h_{0}$ eigenvalues

$$
E_{n l}=\hbar \omega_{h}(2 n+|l|+1)+\frac{1}{2} \hbar \omega_{c} l \pm \frac{1}{2} g \mu_{B} B,
$$

where $\omega_{h}=\sqrt{\omega^{2}+\omega_{c}^{2} / 4}$, and $\omega_{c}=e B / m^{*}$ is the cyclotron frequency. The last term in Eq. (8) (the Zeeman-energy shift is 
taken with plus sign for the spin-up component and with minus for the spin-down component). Below we use a shortened notation $(n, l, s)$, for $s=\uparrow$ or $s=\downarrow$ to describe the contribution of the FD states to the SO wave functions.

The two-electron Hamiltonian

$$
H^{2 e}=H(1)+H(2)+\frac{e^{2}}{4 \pi \epsilon \epsilon_{0} r_{12}},
$$

with GaAs-dielectric constant $\epsilon=12.9$, is diagonalized in a basis of antisymmetrized products of the single-electron eigenfunctions of operator (1),

$$
\Psi=\sum_{\mu, \nu>\mu} d_{\mu, \nu} \chi_{\mu \nu}(1,2),
$$

with $\chi_{\mu \nu}(1,2)=\frac{1}{\sqrt{2}}\left[\psi_{\mu}(1) \psi_{\nu}(2)-\psi_{\mu}(2) \psi_{\nu}(1)\right]$. In the present paper we use all the basis functions that can be constructed of the single-electron basis for FD states with $n<2$ and $|l|$ $\leq 10$. When constructing the two-electron basis for a single type of SO coupling present, it is useful to exploit the conservation of the two-electron total angular momentum by the Coulomb interaction. The eigenstates of Eq. (9) have a definite angular momentum $J=J(1)+J(2)$. The Coulomb interaction conserves also the $s$-parity symmetry, hence the twoelectron stationary states are also eigenstates of the twoelectron $P_{s}(1) \times P_{s}(2)$ operator.

The problem of the single-electron problem can be solved numerically using a few alternative methods. In particular we verified the results presented below for the single-electron problem using a finite-difference imaginary time technique in which the eigenstates of SO coupling Hamiltonian are found without any assumptions on the form of the variational basis and in which an arbitrary precision may be achieved. The basis of FD spin-orbitals-which naturally needs to be truncated-was in fact introduced in order to solve the twoelectron problem. The single-electron functions as given by the finite-difference technique are defined on a mesh of points, which rather excludes the treatment of the twoelectron interaction integrals with a precision necessary to describe the fine effects of the spin-orbit coupling. For that reason we chose to work in a basis of Fock-Darwin spinors given with analytical expressions. Moreover, the stable and fast integration of the Coulomb-matrix elements for the Fock-Darwin basis is widely discussed in the existing literature. In the present paper the integration is performed following the procedure explained in Ref. 31. The truncated basis sets the limit to the maximal angular momentum that can be described with a satisfactory precision. Due to the groundstate angular momentum transitions present in the twoelectron problem, the truncation of the basis limits the range of the magnetic field which can be covered by the present study. In the absence of SO coupling the quality of the variational basis can be verified against the results obtained with separation of the center of mass. The variational overestimate for the ground-state energy as obtained for the truncated basis at $B=15 \mathrm{~T}$ (ground-state angular momentum $l=-9$ ) equals $0.68 \mu \mathrm{eV}$. The corresponding values for $B$ $=16,18,20$, and $23 \mathrm{~T}$ (ground-state $l=-10,-11,-12,-14$ ) are $0.69,1.7,5.2$, and $20 \mu \mathrm{eV}$, respectively. In the present paper we limit our attention to two-electron states in the magnetic fields up to $16 \mathrm{~T}$, which is enough to describe the problem of suppression of one the types of SO coupling by the Zeeman effect.

It is sometimes useful ${ }^{32}$ to express the basis functions or the two-electron wave function in a vector form with each component corresponding to different orientations of the spins of the first and second electron,

$$
\chi_{\mu \nu}(1,2)=\left(\begin{array}{c}
\psi_{\mu}^{\uparrow}(1) \psi_{\nu}^{\uparrow}(2) \\
\psi_{\mu}^{\uparrow}(1) \psi_{\nu}^{\downarrow}(2) \\
\psi_{\mu}^{\downarrow}(1) \psi_{\nu}^{\uparrow}(2) \\
\psi_{\mu}^{\downarrow}(1) \psi_{\nu}^{\downarrow}(2)
\end{array}\right) \text { or } \Psi=\left(\begin{array}{c}
\psi_{\uparrow \uparrow}\left(\mathbf{r}_{1}, \mathbf{r}_{2}\right) \\
\psi_{\uparrow \downarrow}\left(\mathbf{r}_{1}, \mathbf{r}_{2}\right) \\
\psi_{\downarrow \uparrow}\left(\mathbf{r}_{1}, \mathbf{r}_{2}\right) \\
\psi_{\downarrow \downarrow}\left(\mathbf{r}_{1}, \mathbf{r}_{2}\right)
\end{array}\right) \text {. }
$$

From the fermion symmetry the exchange of the spatial and spin coordinates of the electrons leads to inversion of the sign of the wave function

$$
P_{12} \Psi=\left(\begin{array}{c}
\psi_{\uparrow \uparrow}\left(\mathbf{r}_{2}, \mathbf{r}_{1}\right) \\
\psi_{\downarrow \uparrow}\left(\mathbf{r}_{2}, \mathbf{r}_{1}\right) \\
\psi_{\uparrow \downarrow}\left(\mathbf{r}_{2}, \mathbf{r}_{1}\right) \\
\psi_{\downarrow \downarrow}\left(\mathbf{r}_{2}, \mathbf{r}_{1}\right)
\end{array}\right)=\left(\begin{array}{c}
-\psi_{\uparrow \uparrow}\left(\mathbf{r}_{1}, \mathbf{r}_{2}\right) \\
-\psi_{\uparrow \downarrow}\left(\mathbf{r}_{1}, \mathbf{r}_{2}\right) \\
-\psi_{\downarrow \uparrow}\left(\mathbf{r}_{1}, \mathbf{r}_{2}\right) \\
-\psi_{\downarrow \downarrow}\left(\mathbf{r}_{1}, \mathbf{r}_{2}\right)
\end{array}\right),
$$

where $P_{12}$ is the electron exchange operator. In Sec. III for the discussion of the spatial symmetry of the wave function, we consider an operator $R$ which exchanges only the spatial-and not the spin-electron coordinates

$$
R\left(\begin{array}{c}
\psi_{\uparrow \uparrow}\left(\mathbf{r}_{1}, \mathbf{r}_{2}\right) \\
\psi_{\uparrow \downarrow}\left(\mathbf{r}_{1}, \mathbf{r}_{2}\right) \\
\psi_{\downarrow \uparrow}\left(\mathbf{r}_{1}, \mathbf{r}_{2}\right) \\
\psi_{\downarrow \downarrow}\left(\mathbf{r}_{1}, \mathbf{r}_{2}\right)
\end{array}\right)=\left(\begin{array}{c}
\psi_{\uparrow \uparrow}\left(\mathbf{r}_{2}, \mathbf{r}_{1}\right) \\
\psi_{\uparrow \downarrow}\left(\mathbf{r}_{2}, \mathbf{r}_{1}\right) \\
\psi_{\downarrow \uparrow}\left(\mathbf{r}_{2}, \mathbf{r}_{1}\right) \\
\psi_{\downarrow \downarrow}\left(\mathbf{r}_{2}, \mathbf{r}_{1}\right)
\end{array}\right) .
$$

In the absence of SO coupling the two-electron Hamiltonian eigenstates can be separated into products of spin and spatial wave functions. The spatial wave functions are eigenstates of the spatial exchange operator $R$. The spatial wave functions for spin singlets are symmetric (+1 eigenvalue of $R$ ) and for spin triplets they are antisymmetric (-1 eigenvalue of $R$ ) with respect to the exchange of the spatial electron coordinates. The ground-state wave function in the presence of SO coupling is no longer an eigenstate of the $R$ operator. The mean value of the $R$ approaches $1(-1)$ for eigenstates with a dominant singlet (triplet) contribution to the wave function. Deviation of $|\langle R\rangle|$ from unity can be considered as a measure of the nonseparability of the spin and spatial degrees of freedom.

\section{SINGLE ELECTRON STATES}

\section{A. Single type of SO coupling, no Zeeman effect}

In the absence of the Zeeman effect the energy spectra for pure Rashba and pure Dresselhaus couplings are identical. Moreover, for both types of SO coupling, the corresponding energy levels of both spectra are labeled with the same values of the total angular momentum quantum number $J=J_{+}$ for the Rashba coupling and $J=J_{-}$for the Dresselhaus cou- 


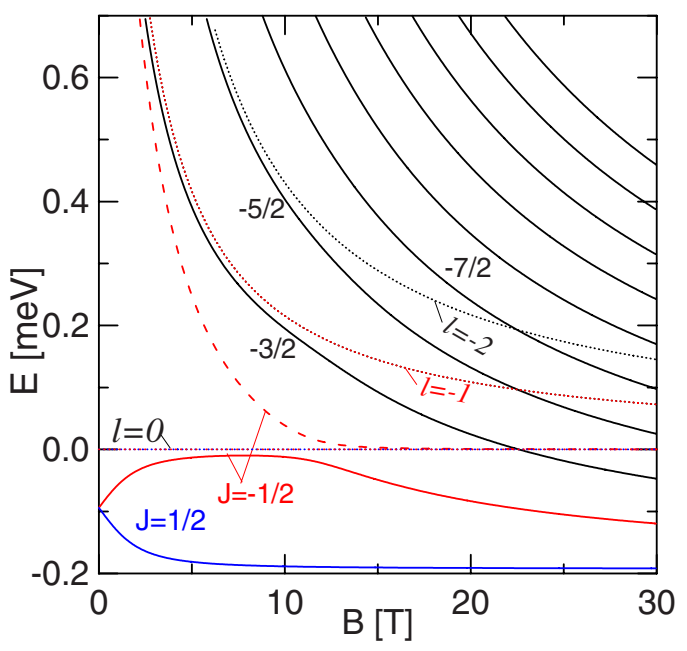

FIG. 1. (Color online) Solid curves show the lowest-energy levels corresponding to the total angular momentum quantum numbers $J=1 / 2,-1 / 2,-3 / 2, \ldots$ calculated for $g=0$ and with only a single type of SO coupling present with coupling constant of $c$ $=10.8 \mathrm{meV} \mathrm{nm}(\alpha=c, \beta=0$ or equivalently $\alpha=0, \beta=c)$. The energy levels are calculated with respect to the lowest-energy FD level. For $J=-1 / 2$ we plot also the second-energy level (dashed curve). The dotted curves show the FD energy levels for $l=0,-1$, and -2 and $n=0$.

pling. For the same quantum number $J$ the eigenstates of $J_{+}$ and $J_{-}$correspond to the same energy and charge density. The spin density for $J_{+}$can be obtained from the $J_{-}$density by inversion.

The lowest-energy levels corresponding to $J=1 / 2$, $-1 / 2,-3 / 2$, etc., are plotted in Fig. 1 with respect to the lowest FD state, i.e., with respect to the ground-state energy in the absence of the SO coupling. The coupling constant $c$ $=10.8 \mathrm{meV} \mathrm{nm}$ is used - the results for the Rashba coupling are obtained for $\alpha=c, \beta=0$ and the ones for the Dresselhaus coupling for $\alpha=0, \beta=c$. For $J=-1 / 2$ in Fig. 1 we additionally plotted the second-energy level, which becomes the ground state when the Zeeman effect is included (see below). Note that the energy of the second $J=-1 / 2$ state tends at high field to the FD ground state (the ground state in the absence SO coupling). The $J=1 / 2$ ground-state energy level saturates at higher $B$ and consequently the SO coupling energy defined as the difference of the ground-state energies with and without SO coupling tends to a constant in the high magnetic-field limit. In the Appendix we demonstrate that SO coupling energy in the high magnetic-field limit is approximately equal to $-2 c^{2} m^{*} / \hbar^{2}$, while the SO coupling at $B=0$ is half smaller, as given by the numerical results of Fig. 1 .

We analyzed contributions of the FD states to the lowestenergy $J= \pm 1 / 2$ states. The results are schematically given for the Dresselhaus type of coupling in Fig. 2 which contains a zoom of the low-energy part of the spectrum of Fig. 1 with the labels of FD configurations $(n, l, s)$ which have dominant contribution for the SO eigenstates. Size of the label indicates relative importance of the contribution. ${ }^{33}$ In order to explain the separate contributions it is useful to look at the $z$ components of the spin given in Fig. $3 .^{34}$ The $J=1 / 2$ ground

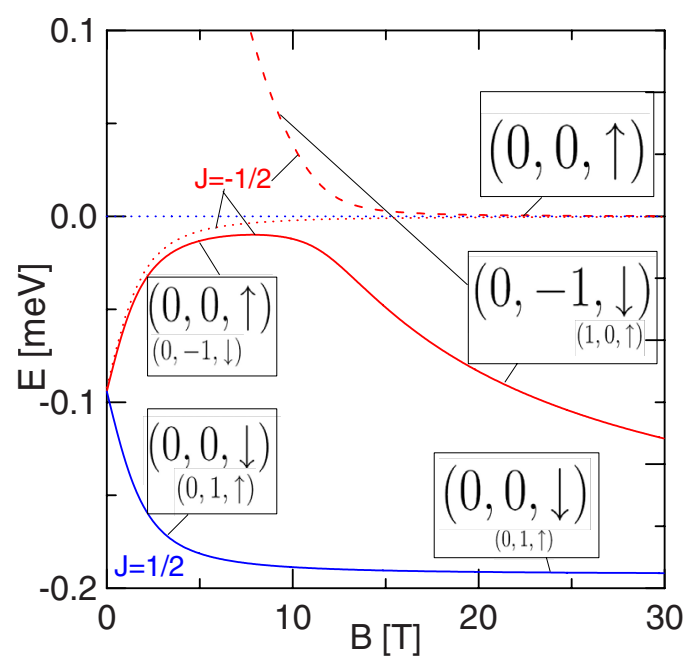

FIG. 2. (Color online) Zoom of the low-energy part of Fig. 1 where we additionally plotted (dotted curves) the lowest-energy $J$ $= \pm 1 / 2$ levels calculated in a basis where only the lowest Landau levels $(n=0$ and $l \leq 0)$ are included. Main contributions of the FD states to the lowest-energy $J= \pm 1 / 2$ states of Fig. 1 are given when only the Dresselhaus coupling is present. We use $(n, l, s)$ notation, where $n$ is the main quantum number, $l$ is the orbital momentum, and $s=\uparrow \downarrow$ is the orientation of the spin. The size of labels $(n, l, s)$ for each energy level indicates the importance of the FD contributions.

state is mainly a superposition of $(0,0, \downarrow)$ and $(0,1, \uparrow)$ states. In particular at $0 \mathrm{~T}$ the FD contributions to the $\mathrm{SO}$ ground state are $95.54 \%$ for $(0,0, \downarrow)$ and $4.4 \%$ for $(0,1, \uparrow)$, while at $50 \mathrm{~T}$ the values are $99.79 \%$ and $0.2 \%$, respectively. We see that the $(0,0, \downarrow)$ FD state in the $J=1 / 2$ SO ground state becomes even more dominant with increasing magnetic field (see Fig. 3). Nevertheless the contribution of the other FD state remains nonzero at high field (see the inset of Fig. 3). The remaining contribution of a spin-up state is necessary to

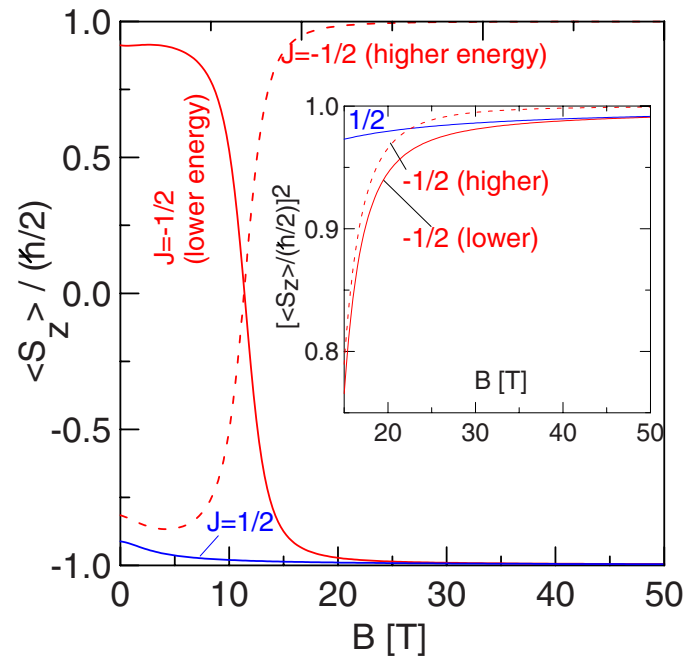

FIG. 3. (Color online) Average $z$ component of the spin for $J= \pm 1 / 2$ energy levels in the presence of Dresselhaus coupling. Applied parameters correspond to Figs. 1 and 2. In the inset square of the average $z$ component of the spin is plotted (the values are identical for Rashba and Dresselhaus types of coupling). 
maintain the SO coupling including the downshift in the energy with respect to the FD ground-state energy level (see Fig. 1). At $B=0$ the lowest-energy $J=-1 / 2$ is degenerate with the $J=1 / 2$ ground-state energy level (Fig. 1) with an inverted mean value of the $z$ component of the spin (Fig. 3). Near $10 \mathrm{~T}$ the two lowest-energy $J=-1 / 2$ levels enter into an avoided level crossing (Fig. 1). At the high field side of the avoided crossing the dominant contribution to the lowerenergy $J=-1 / 2$ state comes from $(0,-1, \downarrow)$ but with an admixture of $(1,0, \uparrow)$ FD state, which stays nonzero even with increasing field (see the inset of Fig. 3-at $50 \mathrm{~T}$ the relative contributions of these two states are $99.77 \%$ and $0.21 \%$, respectively). We find that the second-energy $J=-1 / 2$ state in high $B$ becomes a pure $(0,0, \uparrow)$ FD state and the admixtures of the FD spin-down states disappear much faster than in the two other discussed states [at $50 \mathrm{~T}$ the higher $J=-1 / 2$ state is $99.98 \%$ pure $(0,0, \uparrow)$ FD state]. For that reason the SO coupling effects are totally lifted for the second-energy $J$ $=-1 / 2$ state at high field which leads to the degeneracy with the FD ground-state energy level (see Fig. 1).

The lowest-energy $J=1 / 2$ and $J=-1 / 2$ states only predominantly correspond to the lowest spin-up FD energy branch $(n=0, l \leq 0)$ tending to the lowest Landau level (LLL) in $B \rightarrow \infty$ limit. However, they both contain significant contributions of the spin-down states which preserve the presence of SO coupling also at high field. The SO energy saturated at high $B$. Note that the spin-down states which contribute to the lowest-energy $J= \pm 1 / 2$ states belong to the second Landau level-with $l=1$ for $J=1 / 2$ and with $n=1$ for the lowest-energy $J=-1 / 2$ state. Therefore, of the three states discussed in the context of Figs. 2 and 3 only the second-energy $J=-1 / 2$ at high field can be strictly identified with the lowest Landau level. In Fig. 2 we plotted by the dotted curves the lowest-energy levels corresponding to $J$ $= \pm 1 / 2$ as calculated in the basis limited to the lowest Landau level $(n=0, l \leq 0)$. The limitation of the basis to the lowest Landau level removes completely the SO coupling for $J$ $=1 / 2$. For the lowest-energy $J=-1 / 2$ level the results within the lowest Landau level approximation are qualitatively correct only at low field. Thus we conclude: for pure Rashba and pure Dresselhaus SO coupling description of at least certain lowest-energy states, including the ground state, requires the inclusion of levels beyond the LLL, even at high $B$.

\section{B. Dresselhaus coupling in the presence of the Zeeman effect}

Figure 4 shows the spectrum for pure Dresselhaus coupling with inclusion of the Zeeman effect $(g=-0.44)$. As compared to the $g=0$ case of Fig. 1 , the lowest-energy $J=$ $-1 / 2$ which corresponds mostly to the $(0,0, \uparrow)$ FD state becomes the ground state around $7 \mathrm{~T}$ (see the energy level crossing of $J= \pm 1 / 2$ levels). Figure 4 shows that at the high field the $J=-1 / 2$ ground-state energy becomes equal to the FD ground-energy level. We observed a similar effect for the second-energy $J=-1 / 2$ state for $g=0$ in Fig. 2. For nonzero $g$ this effect is found in the ground state. At $50 \mathrm{~T}$ the ground state is $99.999 \%$ pure $(0,0, \uparrow)$ FD state. In Fig. 4 we notice

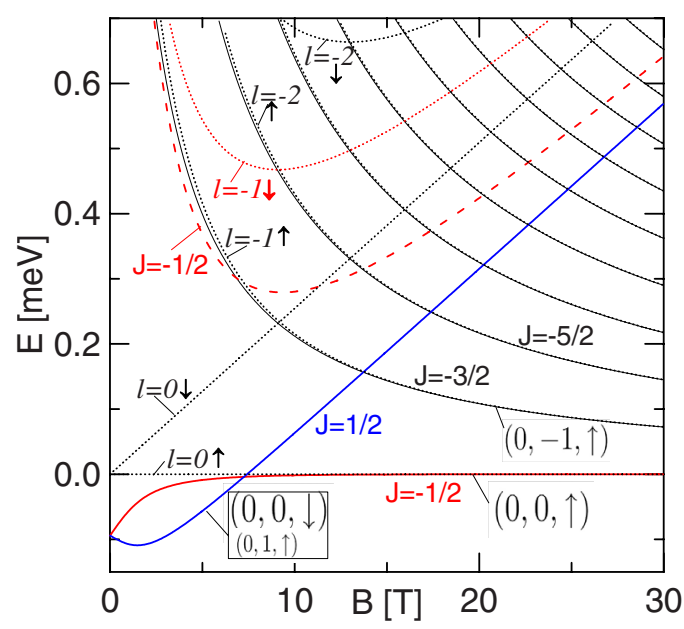

FIG. 4. (Color online) Solid curves show the lowest-energy levels corresponding to the total angular momentum quantum numbers $J=1 / 2,-1 / 2,-3 / 2, \ldots$ calculated when only Dresselhaus coupling with $\beta=10.8 \mathrm{meV} \mathrm{nm}$ is present together with the Zeeman effect with $g=-0.44$. The energy levels are calculated with respect to the lowest-energy FD level. For $J=-1 / 2$ we also show the secondenergy level (dashed curve). The dotted curves show the FD energy levels for a given value of $l=2,1,0,-1,-2, \ldots$. The direction of the spin is indicated for $|l| \leq 2$ FD energy levels. Dominating FD contributions to $J=1 / 2$ energy level as well as to $J=-1 / 2$ and $J=$ $-3 / 2$ at high field are also given.

that also the lowest-energy $J=-3 / 2,-5 / 2, \ldots$ levels can be identified with the spin-up FD energy levels $(n, l, s)=\left(0, J_{-}\right.$ $+1 / 2, \uparrow$ ) of the lowest branch of FD levels (i.e., to LLL) in the high field limit. Therefore, the Zeeman effect at high magnetic field suppresses the Dresselhaus coupling induced mixing of the different spin states of the entire low-energy spectrum. A more analytical insight into suppression of the Dresselhaus coupling effects for the ground state is given in the Appendix, in which we demonstrate that the suppression results from canceling of off-diagonal SO Hamiltonian matrix elements due to the kinetic momentum and the vector potential.

The Zeeman effect has a negligible influence on the wave function of the lowest-energy $J=1 / 2$ state (the energy level plotted as the blue curve in Fig. 4). For $g=-0.44$ the contributions of the $(0,0, \downarrow)$ and $(0,1, \uparrow)$ FD states at $50 \mathrm{~T}$ are $99.785 \%$ and $0.214 \%$, while for $g=0$ the contributions are $99.791 \%$ and $0.208 \%$, respectively.

\section{Rashba coupling in the presence of the Zeeman effect}

The spectrum in the presence of Rashba coupling and the Zeeman effect is shown in Fig. 5. The spectrum is similar to the $g=0$ case of Fig. 1 . The avoided crossing of $J=-1 / 2$ energy levels is shifted to lower field-near $5 \mathrm{~T}$ as compared to $10 \mathrm{~T}$ for $g=0$ (see Fig. 1). At $B=0$ the main contribution to the lowest-energy $J=-1 / 2$ state is the $(0,0, \downarrow)$ FD state which rises in energy faster when the Zeeman effect is present. After the avoided crossing the higher energy $J$ $=-1 / 2$ level can be identified with the lowest-energy FD energy level for the spin-down orientation. In contrast to the 


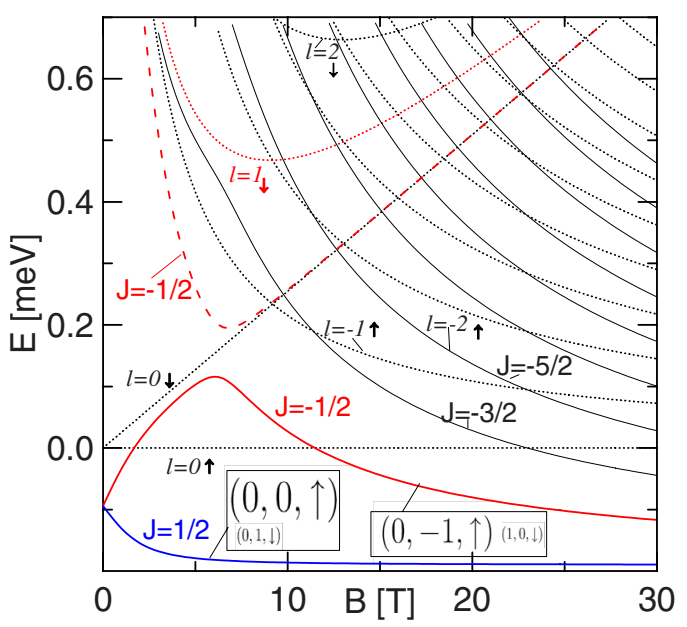

FIG. 5. (Color online) Same as Fig. 4 but now for pure Rashba coupling.

case with Dresselhaus coupling at high magnetic field the lowest-energy levels for a given $J$ do not coincide with the FD spin-up energy levels (dotted lines in Fig. 5). This indicates that the lowest-energy states have nonvanishing spindown admixtures which preserve the presence of the SO energy shift.

\section{Combined Dresselhaus, Rashba, and Zeeman effects}

When both SO couplings are present, at $B=0$ the energy levels are degenerate with respect to the eigenvalues of the $s$-parity operator $P_{s}$. Figure 6 shows the results for $\alpha=\beta$ $=10.8 \mathrm{meV} \mathrm{nm}$. The eigenstate for the lowest-energy odd $s$-parity state which at low field corresponds mainly to $(0,0, \downarrow)$ is shifted up in the energy due to the Zeeman effect.

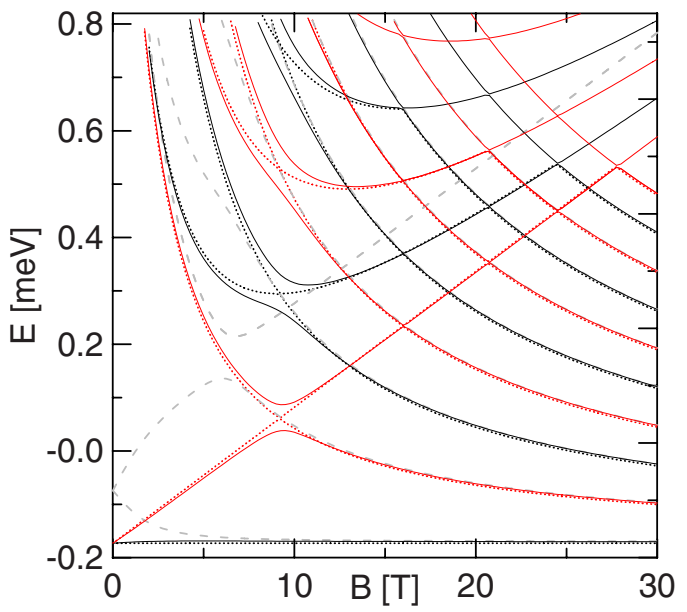

FIG. 6. (Color online) Solid lines show the low-energy spectrum for $\alpha=\beta=10.8 \mathrm{meV} \mathrm{nm}$ and $g=-0.44$ with respect to the lowestenergy FD level in the absence of SO coupling. The red solid (gray solid in print version) and black solid lines correspond to -1 and 1 eigenvalues of $\sigma_{z} P$ operator, respectively. The dotted curves represent the FD energy levels shifted down on the energy scale by $-0.19 \mathrm{meV}$. The dashed curves show the spectrum for pure Rashba coupling.
Near $8 \mathrm{~T}$ this energy level enters into an avoided crossing with another odd $s$-parity state that at low field mainly corresponds to the $(0,-1, \uparrow)$ FD configuration. The dotted curves in the figure show the FD spectrum shifted down by the SO coupling energy $\Delta E=-0.19 \mathrm{meV}$. In Fig. 6 we notice that at the crossings of those FD energy levels that correspond to the same $s$ parity avoided crossings are found in the SO energy spectrum. The number of avoided crossings opened for both coupling present is therefore much larger than in the case of pure Dresselhaus and Rashba couplings where the avoided crossings are formed only at the crossing of FD energy levels which correspond to the same $J_{ \pm}$total angular momentum eigenvalues.

The gray dashed lines in Fig. 6 show the results for pure Rashba coupling. We can see that at high field the lowenergy spectrum for $\alpha=\beta$ becomes identical with the one for $\beta=0$. The effect of the Dresselhaus coupling is therefore suppressed in high magnetic field by the spin Zeeman effect but the Rashba coupling is unaffected.

Outside the avoided crossings the SO levels remarkably coincide with the shifted FD spectrum which indicates a nearly constant SO coupling energy for all the states (this is a specific feature of the $\alpha=\beta$ case). In the Appendix we demonstrate that for pure Rashba or pure Dresselhaus couplings the high magnetic-field limit of the ground-state SO coupling energy is twice larger than for $B=0$. For $\alpha=\beta$ at high magnetic field with nonzero $g$ one of the couplings is removed, so the $B \rightarrow \infty$ limit of SO coupling energy is equal to the case of pure Rashba or pure Dresselhaus coupling. However, at $B=0$ both couplings contribute equally to the SO coupling energy, hence the limits of low and high magnetic field of the SO coupling energy of $\alpha=\beta$ case are equal.

\section{TWO-ELECTRON STATES}

\section{A. Results without Zeeman effect}

Figure 7 shows the two-electron spectrum calculated for a single type of SO coupling present $(\alpha=0, \beta=c$ or equivalently $\alpha=c, \beta=0$ ) for the coupling constant $c$ $=2.7 \mathrm{meV} \mathrm{nm}$ and the Zeeman effect neglected. Energy levels with a single type of SO coupling can be labeled by the total angular momentum $J$ quantum number (see Fig. 7). In the absence of SO coupling the confined electron systems in an external magnetic field undergo ground-state orbital angular momentum transitions. ${ }^{19,22,35}$ As long as $g=0$, the groundstate angular momentum takes on subsequent nonpositive values $L=0,-1,-2, \ldots$, for increasing $B$. The ground-state orbital angular momentum without SO coupling is plotted as the solid curve at the bottom of Fig. 7 . Odd $L$ correspond to spin triplets and the even angular momenta to spin singlets. The mean value of $\langle L\rangle$ in the presence of SO coupling with $c=2.7 \mathrm{meV} \mathrm{nm}$ is shown in Fig. 7 by the dotted curve. SO coupling lifts the degeneracy of the triplets. Each triplet level is split by the SO coupling and the lowest energy one corresponds to the lowest absolute value of $J$. Moreover, the ground state corresponds always to an even value of $J$.

In the angular momenta $L$ ladder without SO coupling (see Fig. 7) we find steps corresponding to even and odd integers which have nearly equal lengths. The SO coupling 


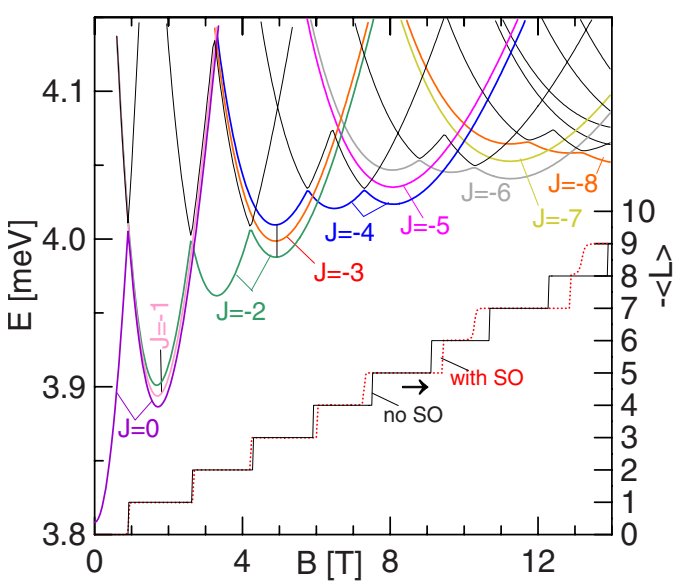

FIG. 7. (Color online) Low-energy spectrum for two-electron states in the presence of a single type of spin-orbit coupling (Rashba or Dresselhaus) with coupling constant $c=2.7 \mathrm{meV} \mathrm{nm}$ ( $\alpha=c, \beta=0$ or $\alpha=0, \beta=c$ ) and $g=0$. The stepwise curves, referred to the right axis, show the ground-state angular momentum. The solid (dotted) line corresponds to the case without (with) SO coupling. Energy levels are calculated with respect to twice the FD ground-state energy.

enlarges the stability range of the states that are nearly antisymmetric with respect to the exchange of spatial coordinates of the electrons (the steps corresponding to odd values of $\langle L\rangle$ are enlarged). Separate Rashba and Dresselhaus couplings promote spin polarization of the ground state but in opposite directions. In Fig. 7 we can also see that the splitting of subsequent ground-state triplets by the SO coupling increases with magnetic field. This effect eventually leads to the removal of those states from the ground state that correspond to even value of $L$. The ground-state polarization induced by the Dresselhaus (Rashba) couplings with spins antiparallel (parallel) to the external magnetic-field vector is illustrated in Fig. 8.

Figure 9 shows the spectrum for equal Dresselhaus and Rashba coupling constants $\alpha=\beta=10.8 \mathrm{meV} \mathrm{nm} \mathrm{for} g=0$. In this case $P_{s}$ is a good quantum number while $J$ is no longer. For a single type of coupling both $J$ and $P_{s}$ quantum numbers are relevant and related by $P_{s}=(-1)^{J+1}$. Red curves (dark gray in the print version) in Fig. 9 correspond to the odd $s$-parity levels and the dashed curves to the even $s$-parity levels. The spectrum when we neglect the SO coupling is plotted with the gray dotted lines and is referred to the right axis. Note that the spectra with and without SO coupling nearly coincide up to a constant energy shift. The SO coupling energy is similar for all the states. The SO coupling results in the opening of avoided crossings between energy levels which cross in the shifted FD spectrum. The inset of Fig. 9 shows a zoom of the avoided crossing marked by the dashed circle in the main panel. We can see that the degeneracy of the triplet energy levels which was split by a single type of SO coupling is restored outside the avoided crossings. This restoration is only observed for $\alpha=\beta$.

Figure 10 shows the wave functions (real part) calculated for four lowest-energy levels at $B=0$ with parameters used above for Fig. 9. The plots are drawn for both electrons situated at the $x$ axis $\left(y_{1}=y_{2}=0\right)$, as function of $x_{1}$ and $x_{2}$.

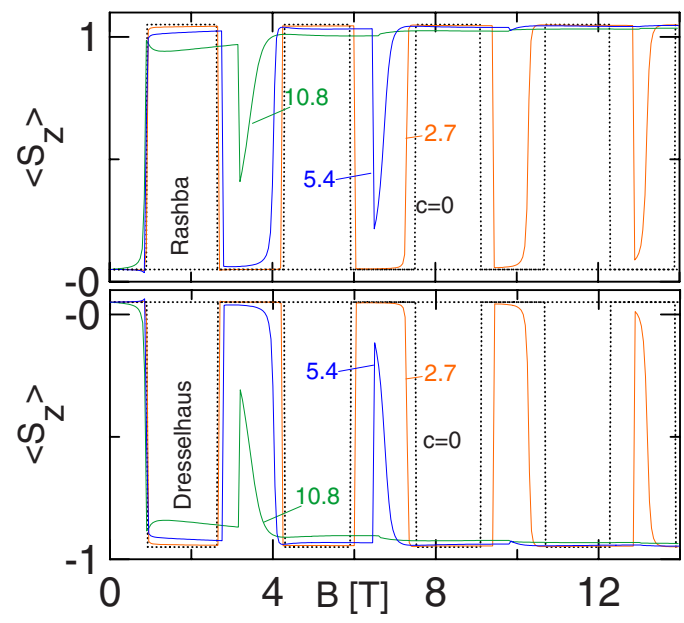

FIG. 8. (Color online) Mean value of the $z$ component of the total spin for the ground state at various coupling constants $c$ (given in $\mathrm{meV} \mathrm{nm}$ ) for a single type of SO coupling present and $g=0$. The dotted curves are for the ground state without SO coupling. The lower panel corresponds to pure Dresselhaus coupling and upper one to pure Rashba coupling.

The subsequent columns of plots in Fig. 10 correspond to the four components of the two-electron wave function $\Psi$ [see Eq. (11)]. We notice that each of the spin-polarized components $\left(\psi_{\uparrow \uparrow}\right.$ and $\left.\psi_{\downarrow \downarrow}\right)$ changes sign at the diagonal of the plot $\left(x_{1}=x_{2}\right)$, which is a result of the first and fourth components of the vector Eq. (12) and is due to the fermion symmetry. The second and third components of Eq. (12) indicate that a plot for each of the spin-unpolarized wave-function components $\left(\psi_{\uparrow \downarrow}\right.$ and $\left.\psi_{\downarrow \uparrow}\right)$ can be obtained from the plot of the other by exchange of the spatial coordinates (inversion with re-

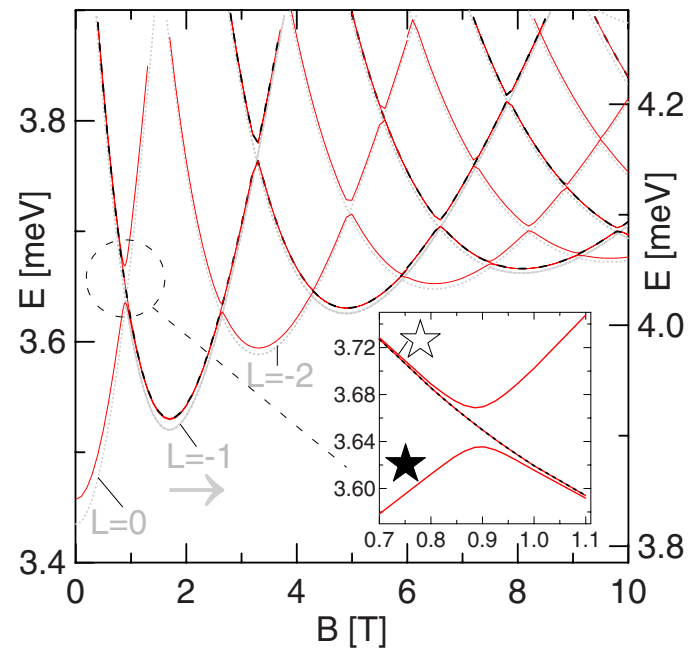

FIG. 9. (Color online) Solid red and dashed curves show the odd and even $s$-parity energy levels for the two-electron states in the presence of both types of SO coupling with $\alpha=\beta=10.8 \mathrm{meV} \mathrm{nm}$ and in the absence of the Zeeman contribution. Energy levels are calculated with respect to twice the FD ground-state energy. The gray dotted lines (referred to the right axis) show the spectrum without the SO coupling. Some of these energy levels are labeled by the angular momentum $L$ quantum number. The inset shows the zoom of the avoided crossing marked by the dashed circle. 


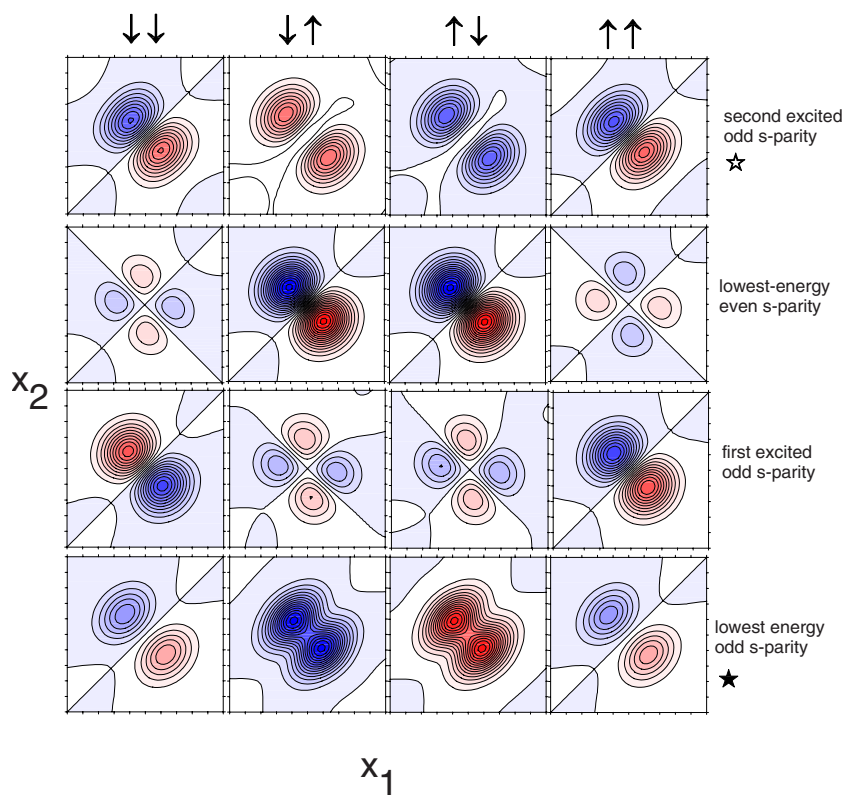

FIG. 10. (Color online) Real parts of the components of the two-electron wave function [see Eq. (11)]. The contours were plotted for $y_{1}=y_{2}=0$ in the $\left(x_{1}, x_{2}\right)$ plane for $\alpha=\beta=10.8 \mathrm{meV} \mathrm{nm}$ at $B=0$. Blue (red) regions correspond to positive (negative) values of the wave function. The rows correspond to states with increasing energy from the bottom to the top and the columns to components of the two-electron wave function indicated by arrows at the top of the plot. The stars mark the states that enter into the avoided crossing shown in the inset of Fig. 9.

spect to the diagonal $x_{1}=x_{2}$ ) followed by the wave-function sign inversion, in accordance with Fig. 10 . The $B=0$ groundstate wave function (the lowest row of plots in Fig. 10) has the $\uparrow \downarrow$ and $\downarrow \uparrow$ components which are similar as in the singletlike case but it also contains both the spin-polarized components. The avoided crossing, shown in the inset of Fig. 9, leads to an energy gap opening between the states marked by stars in Fig. 10. Wave functions for the anticrossing energy levels have the $\uparrow \downarrow$ and $\downarrow \uparrow$ components of the similar singletlike form.

Let us now turn our attention to the spatial exchange operator $R$ of Eq. (13). The mean value of this operator is plotted in Fig. 11 for several choices of the coupling constants (the values for no SO coupling are plotted with the dotted lines). For equal Dresselhaus and Rashba coupling constants and $g=0$ the mean value of $R$ shows oscillations with a period nearly independent of the coupling strength. For $\alpha \neq \beta$ oscillations of $\langle R\rangle$ disappear at high field (see the dashed line) which is related to the approximate spin polarization of the system which occurs due to domination of one of the types of the SO coupling. Figure 12 shows the groundstate average $z$ component of the spin for $\alpha=0, \beta$ $=10.8 \mathrm{meV} \mathrm{nm}$ (dotted line) and $\alpha=5.4 \mathrm{meV} \mathrm{nm}, \quad \beta$ $=10.8 \mathrm{meV} \mathrm{nm}$ (solid line). The Rashba coupling present for $\alpha=\beta / 2$ tends to prevent the spin-down polarization of the ground state but its effect is weak. Moreover, similarly as explained above for the single-electron states for $g=0$, the ground-state spin polarization at high field is approximate and not complete.

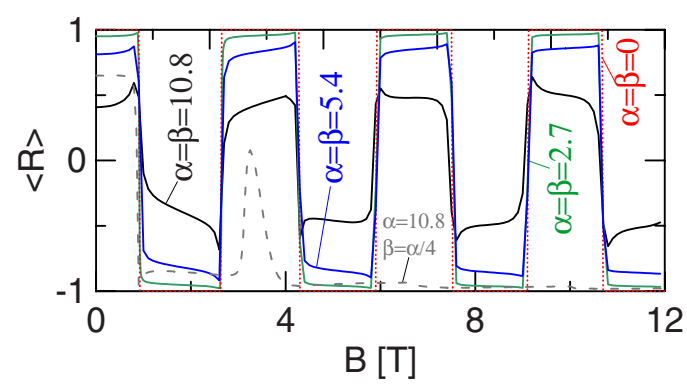

FIG. 11. (Color online) Solid curves show the ground-state expectation values of the interchange of spatial coordinates of electrons for $\alpha=\beta$ and $g=0$. The dotted line shows the values for no SO coupling and the dashed line corresponds to $\alpha=4 \beta$ $=10.8 \mathrm{meV} \mathrm{nm}$ (same result is obtained for $\beta=4 \alpha$ $=10.8 \mathrm{meV} \mathrm{nm}$ )

\section{B. Role of the Zeeman effect for two electrons}

Figure 13 shows the spectrum for pure Dresselhaus coupling with the Zeeman effect present. At high magnetic field the low-energy spectrum becomes identical to the spectrum obtained for no SO coupling (the energy levels for $\alpha=\beta=0$ are plotted by the dotted curves). The Dresselhaus coupling energy is therefore removed from the low-energy spectrum similarly as previously discussed in the single-electron problem.

For pure Rashba coupling (Fig. 14) the SO effect for the energy levels is preserved at high $B$. The (approximate) spin polarization is obtained for distinctly lower field than in the case of Dresselhaus coupling (see the plots of $\left\langle S_{z}\right\rangle$ in Figs. 13 and 14).

The spectrum when both types of SO coupling are active $(\alpha=\beta=10.8 \mathrm{meV} \mathrm{nm})$ is presented in Fig. 15 by the solid curves. At high magnetic field the low-energy spectrum tends to the values obtained for $\beta=0$, and $\alpha=10.8 \mathrm{meV}$, i.e., for pure Rashba coupling - see the dotted lines in the figure.

Figure 16 shows the $z$ component of the spin for pure Rashba, pure Dresselhaus coupling, as well as for equal

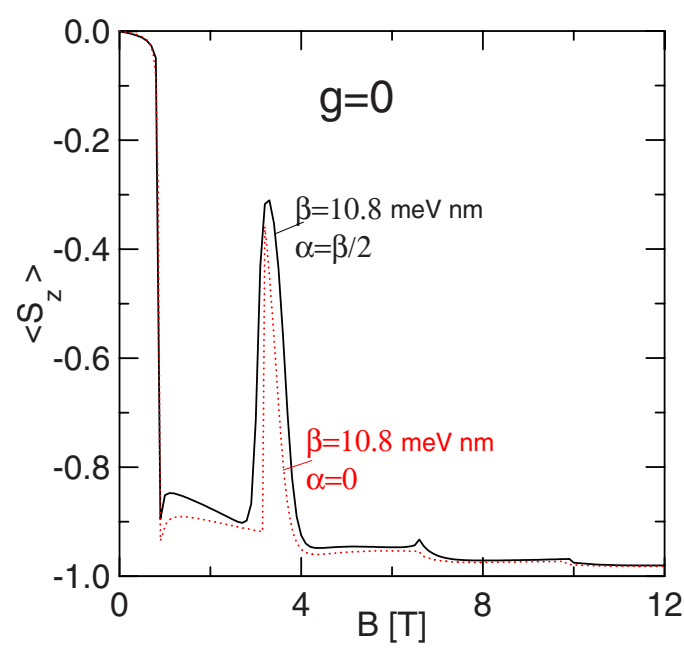

FIG. 12. (Color online) Mean value of the $z$ component of the total spin for the ground state calculated for $g=0$ and pure Dresselhaus SO coupling (dotted curve, $\beta=10.8 \mathrm{meV} \mathrm{nm}, \alpha=0$ ) and dominant Dresselhaus coupling (solid curve, for $\alpha=\beta / 2$ ). 


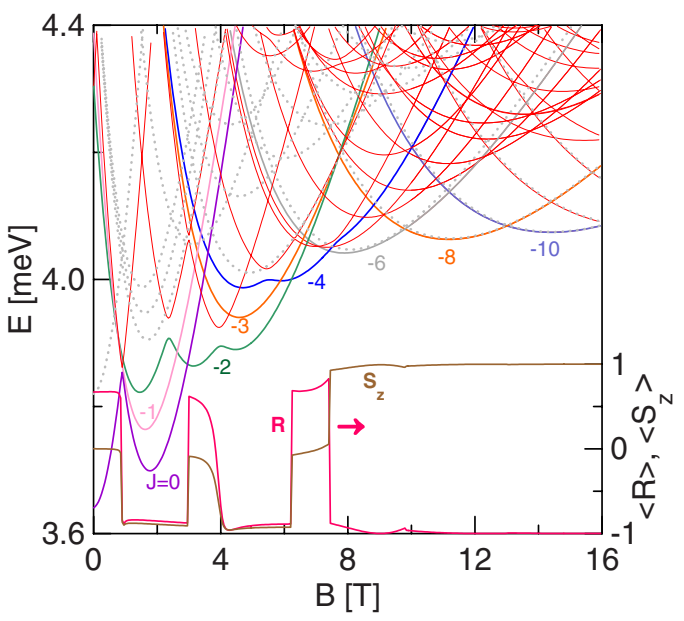

FIG. 13. (Color online) Low-energy spectrum for two-electron states in the presence of pure Dresselhaus coupling for $\beta$ $=10.8 \mathrm{meV} \mathrm{nm}$ including the Zeeman effect $g=-0.44$. Energy levels are calculated with respect to twice the FD ground-state energy. The dotted lines show the energy levels obtained without SO coupling. At the bottom of the plot the average values of the $R$ operator and $\left\langle S_{z}\right\rangle$ for the SO ground state are plotted with respect to the right axis.

Rashba and Dresselhaus coupling constants. The inset shows that the spin-up polarization at high field is strongest for pure Dresselhaus coupling in spite of the fact that the Dresselhaus term itself prefers the spin-down polarization. The polarization is due to the suppression of the Dresselhaus coupling, as indicated by the spectra of Figs. 13 and 15. Due to the suppression of the Dresselhaus type of coupling by high magnetic field the spin obtained for $\alpha=\beta$ becomes identical with the values obtained for pure Rashba coupling. For nonzero $\alpha$ the spin-up polarization at high field is never complete. A complete spin-up polarization would imply removal of the SO coupling energy which stays of the order of $0.4 \mathrm{meV}$ in the high field limit.

Figure 17 shows a comparison of $\langle R\rangle$ for three sets of parameters including the ones applied in Fig. 16. For pure

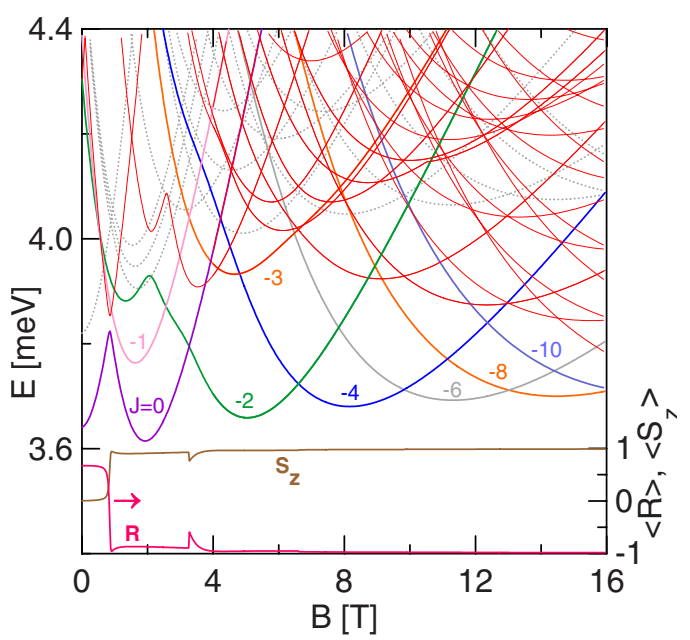

FIG. 14. (Color online) Same as Fig. 13 but now for pure Rashba coupling with $\alpha=10.8 \mathrm{meV} \mathrm{nm}$.

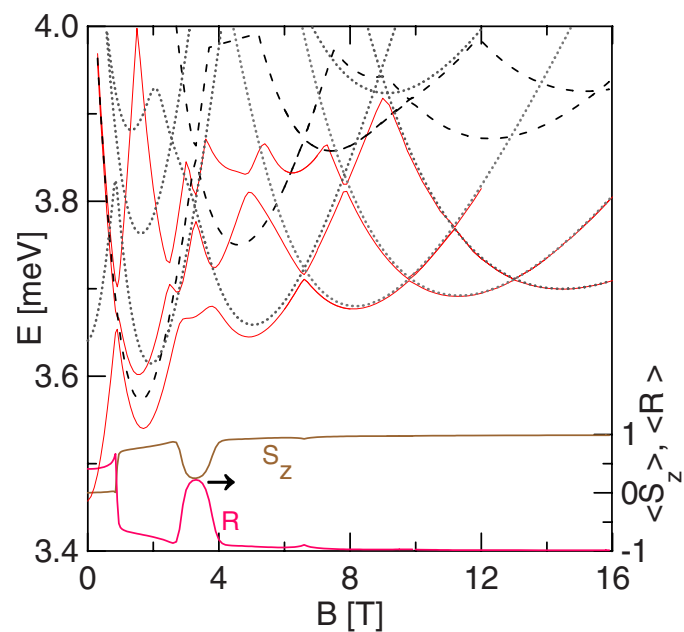

FIG. 15. (Color online) Same as Fig. 13 but for equal Rashba and Dresselhaus SO coupling constants $\alpha=\beta=10.8 \mathrm{meV} \mathrm{nm}$. Energy levels of odd (even) $s$ parity are plotted with solid red (black dashed) lines. Dotted lines show the energy levels obtained for pure Rashba coupling.

Dresselhaus coupling the spin-up polarization of the ground state occurs near $7 \mathrm{~T}$ (see Fig. 16). At the spin flip the ground state has predominantly a singletlike wave function (see the maximum of $\langle R\rangle$ in Fig. 17 near $7 \mathrm{~T}$ ). For higher field the average $R$ value becomes close to -1 in all the considered cases. The zoom in the inset shows that the spatial antisymmetry of the ground state at high field is nearly perfect only for $\alpha=0$, which corresponds to the suppression of the Dresselhaus coupling. The values obtained for $\alpha=\beta$ become equal to the ones obtained for pure Rashba coupling.

Calculations of the properties of few-electron quantum dots at high fields are often performed within the LLL approximation. ${ }^{20,21,23,31}$ Suppression of the Dresselhaus coupling by the Zeeman effect at high magnetic field has another

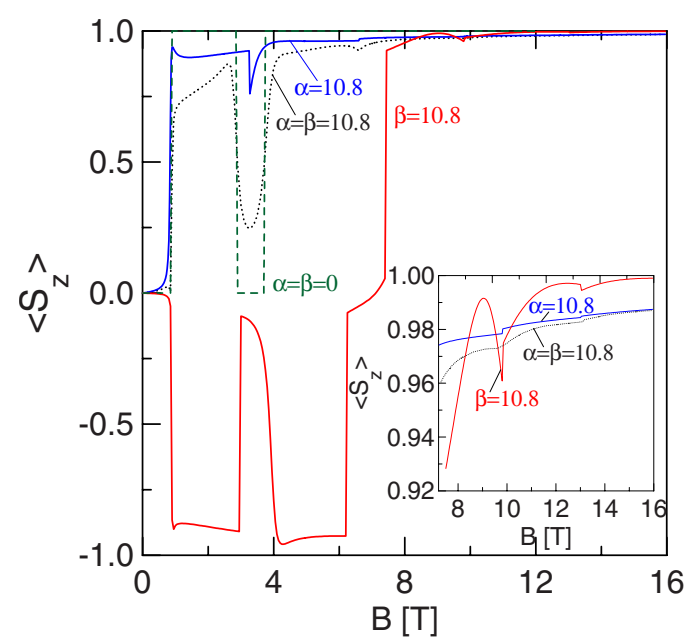

FIG. 16. (Color online) $z$ component of the spin for pure Rashba coupling (blue curve), for pure Dresselhaus coupling (red curve), and for equal Rashba and Dresselhaus coupling constants $\alpha=\beta$ $=10.8 \mathrm{meV} \mathrm{nm}$ (dotted curve) in the presence of the Zeeman effect $g=-0.44$. Dashed line shows the result for no SO coupling. The inset shows the zoom of the higher magnetic-field region. 


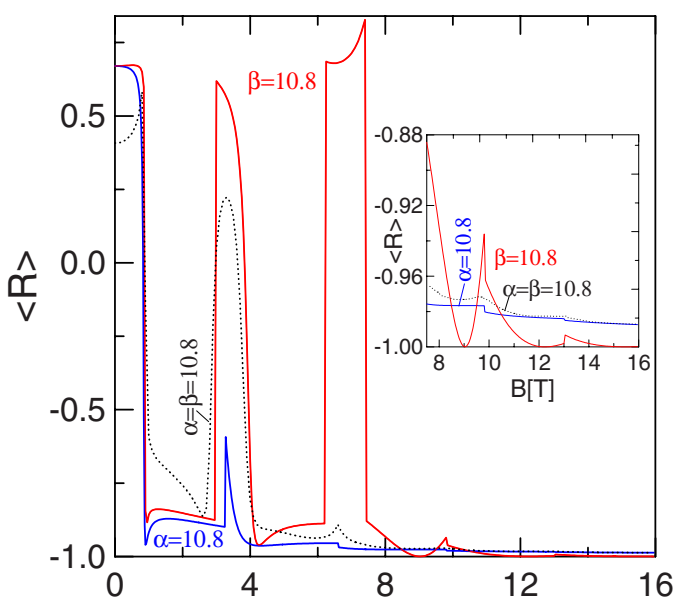

FIG. 17. (Color online) Expectation values of the $R$ operator for pure Rashba coupling (blue line), for pure Dresselhaus coupling (red line), and for equal Rashba and Dresselhaus coupling constants $\alpha=\beta=10.8 \mathrm{meV} \mathrm{nm}$ in the presence of the Zeeman effect $g=$ -0.44 . The inset shows the zoom of the higher magnetic-field region.

consequence. As long as $\alpha=0$ the two-electron ground state at high field can be quite accurately described by the lowest Landau level (lowest FD level) approximation. For $B$ $>10 \mathrm{~T}$ and $\beta=10.8 \mathrm{meV}$, the overestimation of the twoelectron ground-state energy obtained in the LLL approximation is of the order of $0.01 \mathrm{meV}$. On the other hand the LLL is definitely a bad approximation when the Rashba coupling is present. For $\alpha=10.8 \mathrm{meV}$ the energy overestimation obtained in the LLL approximation is of the order of $0.4 \mathrm{meV}$. For nonzero $\alpha$ already the single-electron states cannot be correctly described without inclusion of excited Landau levels (see the results of Figs. 1, 2, and 5).

\section{SUMMARY AND CONCLUSIONS}

We considered stationary states in a parabolic GaAs quantum dot containing a single or two electrons with the inclusion of linear Rashba and Dresselhaus SO coupling terms, for $g=0$ and for the bulk value of the Zeeman term $g$ $=-0.44$. The two-electron problem was solved with the configuration interaction approach.

We analyzed the ground-state spin polarization induced by SO coupling at high magnetic field. In the absence of the Zeeman effect the magnetic field does not suppress the SO coupling and the SO coupling energy saturates at high $B$. As a consequence the ground-state spin polarization is approximate but not complete. Admixture of the contributions with opposite spin orientation to the dominating one is necessary to preserve the SO coupling effects at high field. Similarly, the two-electron wave functions become only approximately antisymmetric with respect to exchange of the electron coordinates.

The strength of the Rashba coupling can be controlled by voltages applied to gates. ${ }^{28,36}$ External electric fields may also influence to some extent the strength of the Dresselhaus coupling by changing the vertical extent of the wave function but they cannot remove it. We demonstrated that Dresselhaus coupling effects are for $g<0$ suppressed at high magnetic field. For $g<0$ and both types of SO coupling present the low-energy spectrum at high $B$ tends to the one obtained for pure Rashba coupling and the total angular momentum $J_{+}$ $=L_{z}+S_{z}$ becomes a good quantum number at high field. For structures without inversion asymmetry the wave functions become exactly antisymmetric with respect to the exchange of the spatial electron coordinates. For $g>0$ the high magnetic field suppresses the Rashba SO coupling from the lowenergy states preserving the Dresselhaus type of coupling.

Approximations based on the lowest Landau level, often used for the description of the few-electron states at high field, are only justified at $g<0$ in the absence of the structural inversion asymmetry.

\section{ACKNOWLEDGMENTS}

This work was supported by the "Krakow Interdisciplinary $\mathrm{PhD}$-Project in Nanoscience and Advanced Nanostructures" operated within the Foundation for Polish Science MPD Programme co-financed by the EU European Regional Development Fund. F.M.P. was supported by the Flemish Science Foundation (FWO-VI) and the Belgian Science Policy (IAP). The work of T. C. was supported by the AGH UST project 11.11.220.01 "Basic and Applied Research in Nuclear and Solid State Physics."

\section{APPENDIX}

The purpose of this appendix is to provide a more analytical insight for suppression of the Dresselhaus coupling that occurs at high magnetic field for $g<0$ in the single-electron ground state. Approximate formulas for the spin-orbit coupling energies for the ground state are also given for both $B=0$ and the high magnetic-field limit.

The results for the single-electron spectra provided in this paper were obtained with a convergent basis of Fock-Darwin orbitals. These results were additionally verified with a nonvariational finite-difference calculation without any assumption on the form of the basis. The results indicate that the lowest-energy $J= \pm 1 / 2$ states can be obtained with a high precision using only $(0,0, \uparrow \downarrow),(1,0, \uparrow \downarrow)$, and $(0, \pm 1, \uparrow \downarrow)$ spin-orbitals. Below we provide results obtained for diagonalization of the SO Hamiltonians in a basis limited to these states.

Let us first consider the $J_{=}-1 / 2$ states for pure Dresselhaus coupling. For three relevant basis states

$$
f_{1}=\left(\begin{array}{c}
\psi_{00} \\
0
\end{array}\right), \quad f_{2}=\left(\begin{array}{c}
\psi_{10} \\
0
\end{array}\right), \quad f_{3}=\left(\begin{array}{c}
0 \\
\psi_{0-1}
\end{array}\right) \text {, }
$$

the Hamiltonian matrix has the form 


$$
H_{D}^{-1 / 2}=\left(\begin{array}{ccc}
\hbar \omega_{h}+\frac{1}{2} g \mu_{B} B & 0 & -\frac{i \beta}{\hbar}\left[\frac{\hbar}{l_{B}}-\frac{1}{2} e B l_{B}\right] \\
0 & 3 \hbar \omega_{h}+\frac{1}{2} g \mu_{B} B & -\frac{i \beta}{\hbar}\left[\frac{\hbar}{l_{B}}+\frac{1}{2} e B l_{B}\right] \\
\frac{i \beta}{\hbar}\left[\frac{\hbar}{l_{B}}-\frac{1}{2} e B l_{B}\right] & \frac{i \beta}{\hbar}\left[\frac{\hbar}{l_{B}}+\frac{1}{2} e B l_{B}\right] & 2 \hbar \omega_{h}-\frac{1}{2} \hbar \omega_{c}-\frac{1}{2} g \mu_{B} B
\end{array}\right) .
$$

Diagonal elements of this matrix are equal to the FD energy levels. In the nondiagonal matrix elements that are due to the SO coupling, the term with the elementary charge results of the contribution of the vector potential to the canonical momentum $[\mathbf{p}=-i \hbar \nabla+e A]$ and the other is due to the kinetic momentum $(-i \hbar \nabla)$. For $B=0$ the lowest eigenvalue of this matrix Hamiltonian is

$$
E_{D}^{-1 / 2}(B=0)=2 \hbar \omega_{0}-\sqrt{\left(\hbar \omega_{0}\right)^{2}+\frac{2 \beta^{2}}{l_{B}^{2}}},
$$

which for the parameters applied in Fig. 2 equals $E_{D}^{-1 / 2}(B=0)=\hbar \omega_{0}-0.094 \mathrm{meV}$ in a good agreement with the full numerical results presented therein.

In the high magnetic-field limit $\omega_{h}=\frac{1}{2} \omega_{c}$ and the Hamiltonian takes the form

$$
H_{D}^{-1 / 2}=\left(\begin{array}{ccc}
\frac{1}{2} \hbar \omega_{c}+\frac{1}{2} g \mu_{B} B & 0 & 0 \\
0 & \frac{3}{2} \hbar \omega_{c}+\frac{1}{2} g \mu_{B} B & -\frac{2 i B}{l_{B}} \\
0 & \frac{2 i B}{l_{B}} & \frac{1}{2} \hbar \omega_{c}-\frac{1}{2} g \mu_{B} B
\end{array}\right),
$$

Note, that the $f_{1}$ spin-up basis state becomes exactly decoupled from the other states, which results from an exact canceling of the kinetic momentum and vector-potential contributions to the matrix elements, since for $\omega_{h}=\omega_{c} / 2$ one has $\frac{1}{2} e B l_{B}=\frac{\hbar}{l_{B}}$. This explains the appearance of the level with energy equal to the Fock-Darwin ground state at high magnetic field

$$
E_{D}^{-1 / 2}(1)=\frac{1}{2} \hbar \omega_{c}+\frac{1}{2} g \mu_{B} B
$$

in which the SO coupling is totally suppressed.

The other two $H_{D}^{-1 / 2}$ eigenvalues for $g=0$ are given by

$$
E_{D}^{-1 / 2}(2 ; g=0)=\hbar \omega_{c}+\frac{\hbar \omega_{c}}{2} \sqrt{1+\frac{8 \beta^{2} m^{*}}{\hbar^{3} \omega_{c}}}
$$

and

$$
E_{D}^{-1 / 2}(3 ; g=0)=\hbar \omega_{c}-\frac{\hbar \omega_{c}}{2} \sqrt{1+\frac{8 \beta^{2} m^{*}}{\hbar^{3} \omega_{c}}}
$$

The second term under the square root in above two formulas is small as compared to 1. Using the Taylor expansion one obtains approximate expressions

$$
E_{D}^{-1 / 2}(2 ; g=0) \simeq \frac{3 \hbar \omega_{c}}{2}+2 \frac{\beta^{2} m^{*}}{\hbar^{2}}
$$

and

$$
E_{D}^{-1 / 2}(3 ; g=0) \simeq \frac{\hbar \omega_{c}}{2}-2 \frac{\beta^{2} m^{*}}{\hbar^{2}} .
$$

For the parameters of Fig. 2 the SO shift of the lower energy of this states $E_{D}^{-1 / 2}(3)$ with respect to the FD ground state equals $-0.19 \mathrm{meV}$. This is the limit value to which the lowest $J=-1 / 2$ energy level tends at high $B$ (see Fig. 2). Note that the high magnetic field-limit of the SO coupling energy is twice larger than the value obtained at $B=0$.

The eigenvector corresponding to $E_{D}^{-1 / 2}(3 ; g=0)$ is very close to $f_{3}$ and the eigenvector for $E_{D}^{-1 / 2}(2 ; g=0)$ is nearly equal to $f_{2}$ (see Sec. III A for the numerical values of contributions of FD states at high field). These eigenvectors are nearly spin polarized. One may then write approximate formulas for nonzero $g$,

$$
E_{D}^{-1 / 2}(2) \simeq \frac{3 \hbar \omega_{c}}{2}+2 \frac{\beta^{2} m^{*}}{\hbar^{2}}+\frac{1}{2} g \mu_{B} B
$$

and

$$
E_{D}^{-1 / 2}(3) \simeq \frac{\hbar \omega_{c}}{2}-2 \frac{\beta^{2} m^{*}}{\hbar^{2}}-\frac{1}{2} g \mu_{B} B .
$$

For $g=0$ at high field the state with suppressed SO coupling $\left[E_{D}^{-1 / 2}(1)\right]$ is the first excited $J=-1 / 2$ energy level (Figs. 1 and 2), while the lowest state corresponds to $E_{D}^{-1 / 2}(3)$. For $g<0$ at high field the order these two eigenvalues is exchanged $E_{D}^{-1 / 2}(1)<E_{D}^{-1 / 2}(3)<E_{D}^{-1 / 2}(2)$. Therefore at high 
field for $g<0$ the lowest-energy $J=-1 / 2$ level is the one with suppressed SO coupling and the energy equal to the ground FD energy level, in agreement with Fig. 4.

For $J=1 / 2$ state an analogous analysis can be performed with flipped spin orientation of the basis elements and inverted sign of the angular momentum

$$
f_{1}=\left(\begin{array}{c}
0 \\
\psi_{00}
\end{array}\right), \quad f_{2}=\left(\begin{array}{c}
0 \\
\psi_{10}
\end{array}\right), \quad f_{3}=\left(\begin{array}{c}
\psi_{01} \\
0
\end{array}\right)
$$

for which the matrix Hamiltonian is

$$
H_{D}^{1 / 2}=\left(\begin{array}{ccc}
\hbar \omega_{h}-\frac{1}{2} g \mu_{B} B & 0 & -\frac{i \beta}{\hbar}\left[\frac{\hbar}{l_{B}}+\frac{1}{2} e B l_{B}\right] \\
0 & 3 \hbar \omega_{h}-\frac{1}{2} g \mu_{B} B & -\frac{i \beta}{\hbar}\left[\frac{\hbar}{l_{B}}-\frac{1}{2} e B l_{B}\right] \\
\frac{i \beta}{\hbar}\left[\frac{\hbar}{l_{B}}+\frac{1}{2} e B l_{B}\right] & \frac{i \beta}{\hbar}\left[\frac{\hbar}{l_{B}}-\frac{1}{2} e B l_{B}\right] & 2 \hbar \omega_{h}+\frac{1}{2} \hbar \omega_{c}+\frac{1}{2} g \mu_{B} B
\end{array}\right) .
$$

For $B=0$ this Hamiltonian is equal to $H_{D}^{-1 / 2}$ which leads to appearance of the Kramers degeneracy of $J= \pm 1 / 2$ energy levels. At high field the lifting of the off-diagonal SO coupling terms occurs for the second basis element, which therefore becomes the eigenstate of the Hamiltonian matrix with energy

$$
E_{D}^{1 / 2}(2)=\frac{3}{2} \hbar \omega_{c}-\frac{1}{2} g \mu_{B} B,
$$

while the other two-energy levels are

$$
\begin{gathered}
E_{D}^{1 / 2}(1) \simeq \frac{\hbar \omega_{c}}{2}-2 \frac{\beta^{2} m^{*}}{\hbar^{2}}-\frac{1}{2} g \mu_{B} B, \\
E_{D}^{1 / 2}(3) \simeq \frac{3 \hbar \omega_{c}}{2}+2 \frac{\beta^{2} m^{*}}{\hbar^{2}}+\frac{1}{2} g \mu_{B} B .
\end{gathered}
$$

For $g=0$ at high field the lowest-energy levels for both $J= \pm 1 / 2$ become degenerate $E_{D}^{1 / 2}(1)=E_{D}^{-1 / 2}(3)$ with the same limit SO coupling energy of $-2 \frac{\beta^{2} m^{*}}{\hbar^{2}} . E_{D}^{1 / 2}(1)$ is the lowest high field $J=1 / 2$ energy level also for $g=-0.44$ in spite of the fact that its spin is nearly oriented antiparallel to the magnetic field. At high field-when the spin Zeeman energy (linear in $B$ ) becomes larger than the SO coupling energy $-E_{D}^{1 / 2}(1)$ exceeds $E_{D}^{-1 / 2}(1)$ and the latter state with lifted SO coupling energy becomes the ground state (Fig. 4).

The same analysis for the Rashba coupling produces the same expressions for the energies only with inverted sign of the Zeeman contributions and $\alpha$ constant replacing $\beta$,

$$
\begin{gathered}
E_{R}^{-1 / 2}(1)=\frac{1}{2} \hbar \omega_{c}-\frac{1}{2} g \mu_{B} B, \\
E_{R}^{-1 / 2}(2) \simeq \frac{3 \hbar \omega_{c}}{2}+2 \frac{\alpha^{2} m^{*}}{\hbar^{2}}-\frac{1}{2} g \mu_{B} B,
\end{gathered}
$$

$$
\begin{gathered}
E_{R}^{-1 / 2}(3) \simeq \frac{\hbar \omega_{c}}{2}-2 \frac{\alpha^{2} m^{*}}{\hbar^{2}}+\frac{1}{2} g \mu_{B} B, \\
E_{R}^{1 / 2}(1) \simeq \frac{\hbar \omega_{c}}{2}-2 \frac{\alpha^{2} m^{*}}{\hbar^{2}}+\frac{1}{2} g \mu_{B} B, \\
E_{R}^{1 / 2}(2)=\frac{3}{2} \hbar \omega_{c}+\frac{1}{2} g \mu_{B} B, \\
E_{R}^{1 / 2}(3) \simeq \frac{3 \hbar \omega_{c}}{2}+2 \frac{\alpha^{2} m^{*}}{\hbar^{2}}-\frac{1}{2} g \mu_{B} B .
\end{gathered}
$$

Lifting of SO coupling at high field occurs for the eigenvalue $E_{R}^{-1 / 2}(1)$ which corresponds to the spin-oriented antiparallel to the magnetic field. The ground state for both $g=0$ and $g$ $=-0.44$ corresponds to $E_{R}^{1 / 2}(1)$ which is stabilized by both the SO coupling and the Zeeman effect. Therefore, lifting of SO coupling occurs not in the ground state but in the first excited $J=-1 / 2$ energy level (see the dashed red curve in Fig. 5 which coincides with the spin-down FD energy level).

To conclude this appendix, we have demonstrated that at high magnetic field both Rashba and Dresselhaus spectra possess a $J=-1 / 2$ energy level with suppressed SO coupling. The suppression of the SO coupling effects appears as a result of lifting of the off-diagonal matrix elements due to an exact cancellation of terms due to the kinetic momentum and the vector-potential contributions to the canonical momentum. For $g=0$ the energy level with suppressed SO coupling is the first excited $J=-1 / 2$ energy level with spinoriented antiparallel (Rashba) or parallel (Dresselhaus) to the magnetic-field vector. When $g<0$ the state with lifted SO coupling occurs as the single-electron ground state only for the Dresselhaus type of coupling since the spin Zeeman interaction-linear in $B$-exceeds the spin-orbit coupling energy which saturates at high $B$. A similar suppression of SO coupling is also obtained for the ground state with Rashba coupling but for $g>0$. 
${ }^{1}$ Y. A. Bychkov and E. I. Rashba, J. Phys. C 17, 6039 (1984).

${ }^{2}$ G. Dresselhaus, Phys. Rev. 100, 580 (1955).

${ }^{3}$ D. Loss and D. P. DiVincenzo, Phys. Rev. A 57, 120 (1998).

${ }^{4}$ S. Bednarek and B. Szafran, Phys. Rev. Lett. 101, 216805 (2008); Nanotechnology 20, 065402 (2009).

${ }^{5}$ P. Foldi, B. Molnar, M. G. Benedict, and F. M. Peeters, Phys. Rev. B 71, 033309 (2005); P. Foldi, O. Kalman, M. G. Benedict, and F. M. Peeters, Nano Lett. 8, 2556 (2008).

${ }^{6}$ K. C. Nowack, F. H. L. Koppens, Yu. V. Nazarov, and L. M. K. Vandersypen, Science 318, 1430 (2007).

${ }^{7}$ N. E. Bonesteel, D. Stepanenko, and D. P. DiVincenzo, Phys. Rev. Lett. 87, 207901 (2001).

${ }^{8}$ D. Bacon, J. Kempe, D. A. Lidar, and K. B. Whaley, Phys. Rev. Lett. 85, 1758 (2000).

${ }^{9}$ E. Ya. Sherman and D. J. Lockwood, Phys. Rev. B 72, 125340 (2005).

${ }^{10}$ D. V. Bulaev and D. Loss, Phys. Rev. B 71, 205324 (2005); M. Florescu and P. Hawrylak, ibid. 73, 045304 (2006); J. I. Climente, A. Bertoni, G. Goldoni, M. Rontani, and E. Molinari, ibid. 76 085305(R) (2007); M. Prada, R. H. Blick, and R. Joynt, ibid. 77, 115438 (2008).

${ }^{11}$ J. M. Sun, T. Dekorsy, W. Skorupa, B. Schmidt, A. Mucklich, and M. Helm, Phys. Rev. B 70, 155316 (2004).

${ }^{12}$ P. Pietiläinen and T. Chakraborty, Phys. Rev. B 73, 155315 (2006).

${ }^{13}$ M. S. Kushwaha, J. Appl. Phys. 104, 083714 (2008).

${ }^{14}$ T. Chakraborty and P. Pietiläinen, Phys. Rev. Lett. 95, 136603 (2005).

${ }^{15}$ M. Valin-Rodriguez, A. Puente, and L. Serra, Phys. Rev. B 69, 153308 (2004).

${ }^{16}$ P. Lucignano, B. Jouault, A. Tagliacozzo, and B. L. Altshuler, Phys. Rev. B 71, 121310(R) 2005.

${ }^{17}$ T. H. Oosterkamp, J. W. Janssen, L. P. Kouwenhoven, D. G. Austing, T. Honda, and S. Tarucha, Phys. Rev. Lett. 82, 2931 (1999).

${ }^{18}$ J. Könemann, R. J. Haug, D. K. Maude, V. I. Fal'ko, and B. L. Altshuler, Phys. Rev. Lett. 94, 226404 (2005).

${ }^{19}$ S. M. Reiman and M. Manninen, Rev. Mod. Phys. 74, 1283 (2002).

${ }^{20}$ C. C. Chang, G. S. Jeon, and J. K. Jain, Phys. Rev. Lett. 94, 016809 (2005).

${ }^{21}$ C. Yannouleas and U. Landman, Phys. Rev. B 69, 113306
(2004).

${ }^{22}$ M. B. Tavernier, E. Anisimovas, F. M. Peeters, B. Szafran, J. Adamowski, and S. Bednarek, Phys. Rev. B 68, 205305 (2003).

${ }^{23}$ R. B. Laughlin, Phys. Rev. Lett. 50, 1395 (1983).

${ }^{24}$ R. C. Ashoori, H. L. Stormer, J. S. Weiner, L. N. Pfeiffer, S. J. Pearton, K. W. Baldwin, and K. W. West, Phys. Rev. Lett. 68, 3088 (1992); J. Kyriakidis, M. Pioro-Ladriere, M. Ciorga, A. S. Sachrajda, and P. Hawrylak, Phys. Rev. B 66, 035320 (2002); J. M. Elzerman, R. Hanson, L. H. Willems van Beveren, L. M. K. Vandersypen, and L. P. Kouwenhoven, Appl. Phys. Lett. 84, 4617 (2004).

${ }^{25}$ C. F. Destefani, S. E. Ulloa, and G. E. Marques, Phys. Rev. B 70, 205315 (2004).

${ }^{26}$ W. Knap, C. Skierbiszewski, A. Zduniak, E. Litwin-Staszewska, D. Bertho, F. Kobbi, J. L. Robert, G. E. Pikus, F. G. Pikus, S. V. Iordanskii, V. Mosser, K. Zekentes, and Yu. B. Lyanda-Geller, Phys. Rev. B 53, 3912 (1996).

${ }^{27}$ B. A. Bernevig, J. Orenstein, and S. C. Zhang, Phys. Rev. Lett. 97, 236601 (2006).

${ }^{28}$ J. D. Koralek, C. Weber, J. Orenstein, A. Bernevig, S. Zhang, S. Mack, and D. Awschalom, Nature (London) 458, 610 (2009).

${ }^{29}$ M. J. Snelling, G. P. Flinn, A. S. Plaut, R. T. Harley, A. C. Tropper, R. Eccleston, and C. C. Phillips, Phys. Rev. B 44, 11345 (1991); M. J. Snelling, E. Blackwood, C. J. McDonagh, R. T. Harley, and C. T. B. Foxon, ibid. 45, 3922 (1992).

${ }^{30}$ W. H. Kuan, C. S. Tang, and W. Xu, J. Appl. Phys. 95, 6368 (2004).

${ }^{31}$ E. Anisimovas and A. Matulis, J. Phys.: Condens. Matter 10, 601 (1998).

${ }^{32}$ S. Moskal, S. Bednarek, and J. Adamowski, Phys. Rev. A 76, 032302 (2007).

${ }^{33}$ In order to obtain the FD configurations that contribute to the eigenstates in the presence of pure Rashba coupling one needs to invert the arrows in Fig. 2.

${ }^{34}$ The results for pure Rashba coupling can be obtained as $2\left\langle S_{z}^{R}\right\rangle$ $=1-2\left\langle S_{z}^{D}\right\rangle$, where $\left\langle S_{z}^{D}\right\rangle$ is the $z$ component of the spin for pure Dresselhaus coupling.

${ }^{35}$ M. Wagner, U. Merkt, and A. V. Chaplik, Phys. Rev. B 45, 1951 (1992); P. A. Maksym, ibid. 53, 10871 (1996).

${ }^{36}$ J. Nitta, T. Akazaki, H. Takayanagi, and T. Enoki, Phys. Rev. Lett. 78, 1335 (1997); D. Grundler, ibid. 84, 6074 (2000). 